\title{
RESEARCH
}

Turkish Journal of Geriatrics

DOI: 10.31086/tigeri.2020.182

2020; 23(4): 446-454

- Burcu TALAY MUSTAFAOĞLU'

- Şule TAŞ GÜLEN ${ }^{1}$ (D)

- Fatih BIRTEKOCAK² (D)

- Aslihan KARUL ${ }^{2}$ D

- Fisun KARADAĞ ${ }^{1}$ (D)

CORRESPONDANCE

'Şule TAŞ GÜLEN

Adnan Menderes University, Faculty of

Medicine, Department of Chest Diseases,

Aydin, Turkey

Phone: +905309943091

e-mail: sule.tas@adu.edu.tr

Received: Oct 09, 2020

Accepted: Nov 19, 2020

Adnan Menderes University, Faculty of

Medicine, Department of Chest Diseases,

Aydin, Turkey

${ }^{2}$ Adnan Menderes University, Faculty of

Medicine, Department of Biochemistry, Aydin,

Turkey

\section{FACTORS AFFECTING FRAILTY SYNDROME IN ELDERLY CHRONIC OBSTRUCTIVE PULMONARY DISEASE PATIENTS AND ITS RELATIONSHIP WITH SYSTEMIC INFLAMMATION}

\section{Abstract}

Introduction: The aim was to identify the factors affecting the frailty syndrome in elderly chronic obstructive lung disease patients and to investigate its relationship with systemic inflammation.

Materials and Method: 61 stable patients (ages of 65-84) were included. Demographic data, body mass index, comorbidities, annual number of exacerbations were recorded. Pulmonary function test, six-minute walk test and Tilburg Frailty Test were performed in all cases. Patients were classified into groups $A, B, C \& D$ according to GOLD chronic obstructive lung disease guideline by annual exacerbation rate and dyspnea score. Neutrophil lymphocyte ratio, high sensitive C-reactive protein, IL-6, 8OH-dG, IL-18 levels were studied in serum.

Results: According to Tilburg test, $63.9 \%$ of the patients were evaluated as frail. There was a relationship between body mass index and Tilburg frailty score ( $p: 0.035)$; body mass index was lower in the frail group. The number of comorbidities was higher in the frail group ( $p: 0.007)$. There was a positive correlation between frailty and the number of drugs used. The frailty score was significantly higher in GOLD group $B \& C$ than in groups $A \& D$ (p: 0.006).

The neutrophil lymphocyte ratio was significantly higher in the frail group (p:0.007).

Conclusion: Frailty is more frequent in chronic obstructive lung disease patients, especially in those with malnutrition and comorbidities and it is associated with a systemic inflammation marker, neutrophil lymphocyte ratio. Routine assessment of frailty in chronic obstructive lung disease outpatients may allow early interventions, including referral to physical and respiratory rehabilitation, geriatric and nutritional specialists to improve physical performance and quality of life of these patients.

Keywords: Frailty; Pulmonary Disease, Chronic Obstructive; Inflammation 


\section{INTRODUCTION}

Aging is described as a perpetual and universal process seen in every living being, leading to a reduction in all bodily functions. This process involves irreversible alterations in structural, functional, mental, and biopsychosocial functions of the organism at the level of molecules, cells, tissues, organs, and systems, manifesting in time (1). Frailty is defined as a geriatric syndrome manifesting with aging, characterized by fatigue and reduction of physiological reserves, and it is affected by various stressful situations. Considering the gradually increasing lifespan, this syndrome has been gaining more importance. Although numerous methods have been implemented for diagnostic assessment, the most commonly used methods are the Fried Scale (frailty phenotype) and the Frailty Index. The Fried Scale involves the parameters of fatigue, reduction in gait speed, reduction in physical activity, weakness, and weight loss, and describes frailty as a clinical syndrome involving three or more of these parameters. The Frailty Index involves a comprehensive geriatric evaluation consisting of cumulative clinical deficits. As these two scales comprise different parametes, there is no consensus on the validity of one over the other $(2,3)$. Another method used for diagnostic assessment is the Tilburg Frailty Scale (TFS). TFS is a brief, simple, and global test that is suitable for use in both daily clinical practice and research; it measures the physical, psychological, and social function losses which are three essential components of frailty. TFS consists of 25 questions, and the validity and reliability of the Turkish form has been proven in the country (4).

Chronic obstructive pulmonary disease (COPD) is a systemic inflammatory disorder with high morbidity and mortality and is characterized by partially irreversible airway constriction $(5,6)$. It is well known that COPD is not just a disease confined to the lung, but also has a clinical course with extrapulmonary manifestations and associated comorbidities related to systemic inflammation $(7,8)$. Frailty syndrome is one of the comorbidities seen in COPD, with prevalence varying between $10-57 \%$. Frailty has common risk factors with COPD, such as age, smoking, and endocrine dysfunction. Hypoxemia and dysfunction of skeletal muscles leading to reduction in cognitive and functional performance, and other phenotypic changes similar to those seen in frailty syndrome, such as fatigue, loss of appetite, weight loss, and decreased physical activity, are observed in patients with COPD. Additionally, recent studies have shown that frailty and related reduction of physical performance are among the significant determinative factors contributing to exacerbations and recurrent hospitalizations in COPD (9-11). In our study, we aimed to investigate factors such as the severity of COPD, frequency of exacerbations, functional assessment, and comorbidities, which may affect frailty syndrome in older patients with COPD, along with the association of frailty syndrome with systemic inflammation and oxidative stress.

\section{MATERIALS and METHODS}

The study was conducted in Adnan Menderes University Hospital, Department of Chest Diseases between June 2018 and October 2019, following the approval of the local ethics committee (protocol \# 53043469-050.04.04). Sixty-one patients aged between 65-84 years, who were diagnosed with COPD and were stable according to the Global initiative for chronic obstructive lung disease (GOLD) 2019 criteria were included in the study, after obtaining their written informed consent (12). Those patients who did not have any acute worsening of symptoms, requiring additional treatment were considered "stable." Patients with a systemic inflammatory disease or malignancy, those within the COPD exacerbation period, and those who did not consent to participate were excluded from the study.

\section{Study Protocol}

Demographic data of all cases consisting of age, gender, occupation, smoking history, body 
mass index (BMI), medical history, and physical examination were recorded. Patients were classified into groups $A, B, C \& D$ according to GOLD COPD guideline by annual exacerbation rate and dyspnea score (12). The additional details included associated comorbidities, names and doses of medications used, number of hospitalizations, exacerbation frequency, six-minute walking test (6MWT) result, COPD assessment test (CAT) result, and the results of Modified Medical Research Council (mMRC) dyspnea scale, and body-mass index, airflow obstruction, dyspnea, and exercise (BODE) Index used for determining the severity of dyspnea. BMl $\leq 21 \mathrm{~kg} /$ $\mathrm{m}^{2}$ was considered low and $\mathrm{BMI}>21 \mathrm{~kg} / \mathrm{m} 2$ was considered normal. Pulmonary function tests (PFTs) and the TFS tests were performed in all cases. Serum samples were obtained for neutrophil-lymphocyte ratio (NLR), high-sensitivity C-reactive protein (hsCRP), interleukin-6 (IL-6), and interleukin-18 (IL18) (as indicators of systemic inflammation), and for 8-hydroxy 2-deoxyguanosine $(8 \mathrm{OH}-\mathrm{dG}$ ) levels (as an indicator of oxidative stress).

\section{Pulmonary Function Test}

Pulmonary function tests were performed in accordance with the American Thoracic Society (ATS) / European Respiratory Society (ERS) standards using the Jaeger Master Scope Spirometer (13). The diagnosis and staging of COPD were performed in accordance with the GOLD 2019 criteria (12).

\section{Functional Assessment}

The levels of dyspnea in participants were graded between 0 and 4 using the mMRC criteria. Exercise capacity was evaluated using the 6MWT in a 30-m long hospital corridor. The maximal distance that the patient could walk for 6 min at normal gait speed was recorded in meters. The BODE index was scored according to $\mathrm{BMI}$, forced expiratory volume in 1st second (FEV1), mMRC dyspnea score, and 6MWT result.

\section{Tilburg Frailty Test}

Tilburg Frailty Score, which consisted of two sections and 25 questions, was applied to the cases. Section A involved potential determinants of frailty, such as sociodemographic characteristics, lifestyle, diseases, experienced events, and living environment. Section B involved 15 questions evaluating frailty components (physical, psychological, and social). Eleven items of TFS had the dual-answer form as "yes" and "no, whereas four items had the triple-answer form as "yes," "sometimes," and "no." The "yes" and "sometimes" answers were graded as one point, and the "no" answers as zero points. The results were scored between 0 to 15 points. Cases with scores $\geq 5$ points were considered frail $(4,14)$.

\section{Biochemical Investigation}

In all cases participating in the study, approximately $5 \mathrm{~mL}$ of blood was obtained and placed in a plain biochemistry tube, and then centrifuged at $3000 \mathrm{rpm}$ for $15 \mathrm{~min}$. The sera were separated and stored at $-80^{\circ} \mathrm{C}$ until the day of the study. The hsCRP, IL-6, IL-18, and $80 \mathrm{H}-\mathrm{dG}$ tests were quantitatively studied using commercial ELISA kits. The test results were calculated using the Bioelisa Reader Elx800. The complete blood count parameters were studied using a BC 6800 Mindray device. For the calculation of NLR, the neutrophil count was divided by the lymphocyte count.

\section{Statistical Analysis}

Statistical evaluation was performed using SPSS (Statistical Package for Social Sciences v.21, IBM Corp., Armonk, NY, USA) statistical analysis software. Categorical measurements, such as sex and frailty groups, were expressed as numbers and percentages. Continuous measurements, such as age, height, and body weight, were expressed as mean \pm standard deviation. Regarding the investigation of the difference between the two groups, the "in- 
dependent samples t-test" was used for variables that were in conformity with a normal distribution, and the "Mann-Whitney U" test was used for variables that were not in conformity with a normal distribution. The "Chi-square" test was used for the analysis of relationships between categorical variables, and the results are presented in tables. The results were evaluated at a $95 \%$ confidence interval and a significance level of $p<0.01$. The "Pearson correlation test" and "two-tailed test" were used for correlations. They were evaluated at significance levels of $p<0.01$ and $p<0.05$, respectively.

\section{RESULTS}

A total of 61 cases, consisting of $43(70.5 \%)$ patients in the 65-74 years age group and $18(29.5 \%)$ patients in the 75-84 years age group, were included in the study. $96.9 \%$ of the cases were males, whereas 3.1\% were females. Among all cases, those with an educational level below high school consisted of $87.1 \%$, whereas those with an educational level of high school or above consisted of $12.9 \%$. While $71.9 \%$ of the cases had quit smoking, $18.1 \%$ were active smokers. A smoking history of over 40 pack-years was present in $62.5 \%$ of the cases. $31 \%$ of the cases had a history of hospitalization once or more in the last year due to exacerbations, with $12.5 \%$ of the cases having two or more exacerbations. In $57.8 \%$ of all cases, two or more comorbidities were present.

The cases were grouped as Group 1 (non-frail group [TFS < 5]) $(36.1 \%)$ and Group 2 (frail group (TFS $\geq 5)$ ] (63.9\%). There was no statistically significant relationship between the two groups with regards to age, gender, marital status, education level, monthly income level, smoking history, exacerbation per year, and the number of exacerbations requiring hospitalization. There were statistically significant differences in $\mathrm{BMI}$ and the number of comorbidities between the two groups ( $p=0.035$ and $p=0.007$, respectively). In Group 2, BMI was lower, and the number of comorbidities was higher (Table 1).
No statistically significant differences were determined between Groups 1 and 2 regarding the values of FEV1 (\% predicted), forced vital capacity (FVC) (\% predicted), and post-bronchodilator FEV1/ FVC. The evaluation of GOLD staging showed that patients classified with GOLD group A, group B, group $C$ and group D were 20 (32.8\%), 21 (34.4\%), 5 (8.2\%), and 15 (24.5\%) patients, respectively. Statistically significant differences were seen among GOLD stages regarding frailty status. The frailty scores of GOLD groups B and C were significantly higher than those of GOLD groups $A$ and $D(p=$ 0.006) (Table 2).

Analysis of mMRC dyspnea scores showed that $4.7 \%$ of the cases were mMRC- $0,35.0 \%$ were mMRC1, 34.4\% were mMRC-2, $18.18 \%$ were mMRC-3, and $4.3 \%$ were mMRC- 4 . While the CAT score was equal to or below 10 in $36.5 \%$ of the cases, it was above 10 in $63.5 \%$. The BODE index score was $0-2$ points in $60 \%, 3-4$ points in $25 \%, 5-6$ points in $5.1 \%$, and $7-10$ points in $5.5 \%$ cases. The comparison of Group 1 and Group 2 showed that, the CAT score was significantly higher in Group 2 than in Group $1(p=0.027)$, and the 6MWT distance percentage was significantly lower in Group 2 than in Group 1 ( $p$ $=0.044)$. However, no statistically significant difference was found between the two groups regarding the BODE index (Table 3).

When Groups 1 and 2 were compared regarding the indicators of systemic inflammation and oxidative stress, it was found that a significant difference was present for NLR ( $p=0.007$ ) which was higher in Group 2. However, no statistically significant differences were found regarding hsCRP, IL-6, IL-8, and $80 \mathrm{H}-d \mathrm{G}$ levels ( $p>0.01$ ) (Table 4).

The correlation analyses among frailty index, demographic data, functional parameters, and the indicators of systemic inflammation and oxidative stress revealed a negative correlation between frailty and body weight ( $p=0.017, r=-0.304)$. The frailty index was positively correlated with the number of comorbidities and the number of drugs used by COPD patients $(p=0.002, r=0.383$; and $p=0.002, r$ 
Table 1. Comparison of Demographic Data of Groups 1 and 2

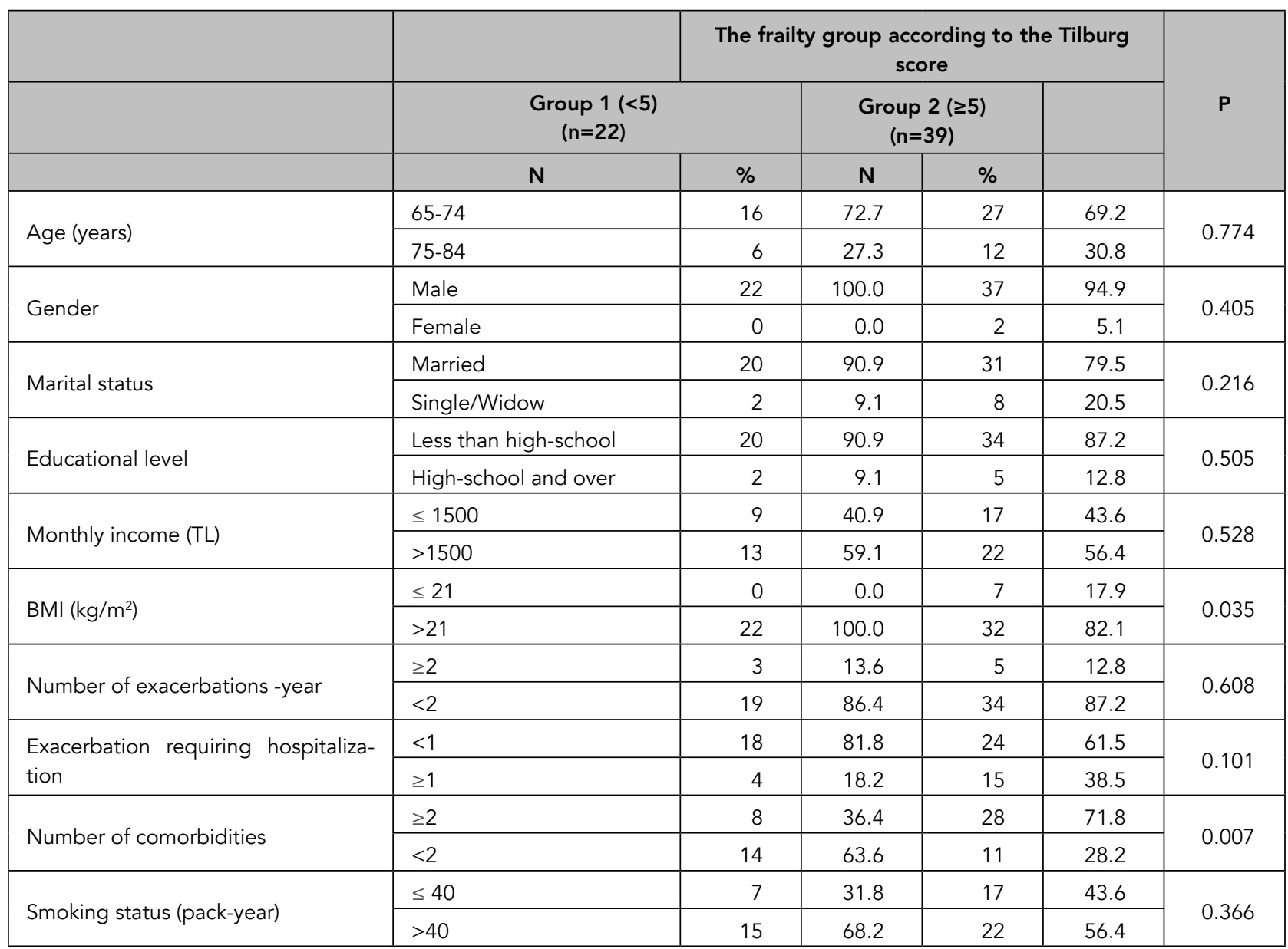

Abbreviations: BMI: body mass index

$=0.390$, respectively). Regarding functional parameters, a positive correlation was present between the CAT score and TFS, and a negative correlation was found between the 6MWT percentage and the TFS $(p=0.005, r=0.360$; and $p=0.019, r=-0.319$, respectively). No correlations were found between the frailty score and the indicators of systemic inflammation and oxidative stress.

\section{DISCUSSION}

In our study, according to the results of the TFS test, $63.9 \%$ of the cases were determined to be frail. Regarding the GOLD staging of COPD, frailty scores of Groups B and D were higher than those of Groups A and D. The frailty score was found to have significant relationships with BMI, the number of comorbidities, the CAT score, 6MWT distance percentage, and NLR, which is one of the indicators of inflammation. Frailty score had a negative correlation with body weight and 6MWT distance percentage, and positive correlations with the CAT score, the number of comorbidities, and the number of drugs used.

Frailty is a geriatric syndrome caused by age-re- 
Table 2. Tilburg Frailty Score According to GOLD staging in COPD

\begin{tabular}{|l|c|c|c|c|c|}
\hline Variable & $\mathbf{A}(\mathbf{n}=\mathbf{2 0})$ & $\mathbf{B}(\mathbf{n}=\mathbf{2 1})$ & $\mathbf{C}(\mathbf{n}=\mathbf{5})$ & $\mathbf{D}(\mathbf{n}=\mathbf{1 5})$ & $\mathbf{P}$ \\
\hline Frailty score & $3.95 \pm 2.13$ & $7.33 \pm 3.45$ & $7.00 \pm 2.65$ & $6.13 \pm 3.05$ & 0.006 \\
\hline Physical Component Score & $2.10 \pm 1.79$ & $4.10 \pm 2.23$ & $4.00 \pm 1.73$ & $3.13 \pm 2.36$ & 0.023 \\
\hline Psychological Component Score & $0.95 \pm 0.92$ & $2.05 \pm 1.32$ & $1.33 \pm 1.53$ & $1.75 \pm 1.34$ & 0.045 \\
\hline Social Component Score & $1.00 \pm 0.45$ & $1.19 \pm 0.60$ & $1.67 \pm 0.58$ & $1.25 \pm 2.00$ & 0.138 \\
\hline
\end{tabular}

Abbreviations: GOLD: Global initiative for chronic obstructive lung disease; COPD: Chronic obstructive pulmonary disease

lated reduction of reserves and functions, leading to decreased ability to cope with daily or acute stress factors. The prevalence of frailty increases with age. Studies have revealed an age related prevalence of $10.7 \%$ in over 65 years, $15.7 \%$ in $80-84$ years, and $26.1 \%$ in over 85 years. Frailty is more commonly detected in females than in males (15). COPD patients who were followed up in our out-patient clinic were mostly males (96.9\%), since COPD is a smoking burden-related disorder; as a result of which an inter-gender comparison could not be made.

Frailty is common in patients with chronic diseases. The prevalence of frailty in COPD patients was reported to be between 6.6-75\% (15). The number of cases and age group that the study was conducted with, differences in methods used, and discrepancies between scales used for identification might be among the causes for such a wide range. In a study conducted by lerodiakonou et al. on COPD patients using the Frail Non-disabled (FIND) survey, frailty was determined with a ratio of $82 \%$ (16). In our study, the prevalence of frailty determined by the TFS test was $63.9 \%$; with the likely causes of such a high prevalence rate, being the screening tool, the number of patients, the study group involving patients over 65 years of age only, and COPD as a disorder with systemic inflammatory effects $(15,17)$.

Most questionnaires used for frailty screening have not been developed to be self-applicable (18). The TFS test is a questionnaire used to determine the frailty status of the patients in our study; it is an understandable and straightforward test that can be used for early diagnosis of health-related prob- lems in the elderly, and its validity and reliability has been proven in Turkey (4).

Chronic diseases are among the critical factors in determining frailty. According to the frailty phenomenon defined by Fried et al., chronic diseases play a significant role in the initiation of frailty (19). The Frailty Index, suggested by Rockwood et al., involves chronic disorders as a criterion of priority (20). Chronic diseases are common risk factors and symptoms associated with frailty. In addition, drugs used in chronic diseases may lead to frailty. In the TFS used in our study, chronic diseases were found to be determinants of frailty. In our study, statistically significant relationships and positive correlations between the number of comorbidities, the number of drugs used, and the TFS were determined, supporting the studies mentioned above.

In a study conducted by lerodiakonou et al., in COPD patients, statistically significant differences were reported between the frail and non-frail

Table 3. Comparison of CAT, BODE Index, and 6MWT Results of Group 1 and 2

\begin{tabular}{|l|c|c|c|}
\hline & $\begin{array}{c}\text { Group 1 } \\
(\mathbf{n = 2 2 )}\end{array}$ & $\begin{array}{c}\text { Group 2 } \\
(\mathbf{n = 3 9 )}\end{array}$ & $\mathbf{P}$ \\
\hline CAT score & $8.64 \pm 5.93$ & $13.39 \pm 7.89$ & 0.027 \\
\hline BODE index & $2 \pm 1.75$ & $2.82 \pm 2.08$ & 0.232 \\
\hline 6MWT (m) & $348.57 \pm 126.59$ & $322.45 \pm 80.68$ & 0.277 \\
\hline 6MWT (\%) & $64.81 \pm 16.45$ & $56.55 \pm 12.87$ & 0.044 \\
\hline
\end{tabular}

Abbreviations: 6MWT: Six-minute walking test, CAT score: COPD evaluation questionnaire 
groups with respect to age, GOLD stage, presence of uncontrolled disease, high CAT and mMRC scores, number of exacerbations, smoking cessation status, and accompanying comorbidities. Similarly, in our study, statistically significant differences were seen between the frail and non-frail patients with respect to the GOLD stage, the CAT score, the number of comorbidities, and the number of drugs used in COPD patients. The frailty score was significantly increased in patients with GOLD stages $B$ and $C$ compared to those in GOLD stages $A$ and $D$. In a study conducted by lerodiakonou et al., the frailty scores of patients in GOLD groups $A$ and $C$ were reported to be significantly higher than those in GOLD groups B and D (16). The reason for such a difference might be the differences in using the $\mathrm{mMRC}$ and CAT scores while determining the groups. In our study, no significant relationship was found between the prevalence of frailty and smoking cessation status, the number of exacerbations, and the mMRC score.

In a study conducted by Kusunose et al., the Kihon checklist score of frailty was found to correlate with the CAT score. In our study, a positive correlation was determined between the TFS and the CAT score, supporting the results of Kusunose's study. In the study by Kusunose et al., no correlation was determined between the total Kihon checklist score and the parameters of the PFTs (9). Similarly, in our study, no correlation was determined between the TFS and the parameters of the PFT.

COPD and frailty have common risk factors such as age and smoking. Besides, they involve common pathophysiological mechanisms such as chronic inflammation, dysregulation of the immune system, and impaired neuroendocrine regulation. Serum levels of inflammatory indicators such as hsCRP, IL6, and TNF-? increase in systemic inflammation (21). In a study conducted by Dogrul et al., investigating the presence of inflammation in patients aged over 65 years, the CRP levels were similar whereas significant differences were reported to be present between frail and non-frail groups with respect to
Table 4. Comparison of Groups 1 and 2 Regarding NLR, and the Indicators of Systemic Inflammation and Oxidative Stress Parameters

\begin{tabular}{|l|c|c|c|}
\hline $\begin{array}{c}\text { Variable } \\
\text { (mean } \pm \text { SD) }\end{array}$ & $\begin{array}{c}\text { Group 1 } \\
(\mathbf{n = 2 2 )}\end{array}$ & $\begin{array}{c}\text { Group 2 } \\
(\mathbf{n = 3 9 )}\end{array}$ & $\mathbf{P}$ \\
\hline $\mathrm{NLR}$ & $2.27 \pm 1.23$ & $3.05 \pm 1.46$ & 0.007 \\
\hline $\mathrm{HsCRP}$ & $1.49 \pm 0.27$ & $1.79 \pm 1.18$ & 0.859 \\
\hline $\mathrm{IL}-6$ & $25.10 \pm 4.77$ & $31.53 \pm 20.82$ & 0.467 \\
\hline $\mathrm{IL}-18$ & $5.26 \pm 3.33$ & $6.24 \pm 4.67$ & 0.912 \\
\hline $8 \mathrm{OH}-\mathrm{dG}$ & $33.90 \pm 9.91$ & $34.19 \pm 6.53$ & 0.554 \\
\hline
\end{tabular}

Abbreviations: NLR: neutrophil lymphocyte ratio, HsCRP: high sensitive C-reactive protein, IL-6: Interleukin-6, IL-18: Interleukin-18, 8OH-dG: 8-Hydroxy 2-deoxyguanosine

sedimentation, NLR, and CRP/albumin ratio (22). Similar to the study of Dogrul et al., a significant difference was determined between the groups with respect to NLR in the present study. However, no significant differences were found between the hs-CRP, IL-6, IL-18, and $80 \mathrm{H}-\mathrm{dG}$ values of the study groups. Besides, no significant correlations between the Tilburg score and the indicators of inflammation and oxidative stress were present in our study. The result that no difference was found between the two groups regarding the indicators of inflammation, except for NLO, might have originated from the fact that most of the patients in both groups were diagnosed as having severe COPD accompanied by systemic inflammation.

In COPD patients, dyspnea leads to immobility, and physical activity decreases with increasing age. Walking distance, which is an important determinant of frailty and showing exercise capacity, is an essential parameter reflecting the multi-systemic effects of disease severity in COPD. Walking distance can be evaluated using the 6MWT (23). Few studies investigating frailty and walking tests are available in the literature. In a study conducted by Gale et al., the 6MWT result of the frail group was reported to be significantly lower than that of the non-frail group. In addition, a negative correlation was reported between the 6MWT and frailty (24). 
Similarly, a negative correlation was found between the 6MWT distance percentage and frailty score in our study.

In COPD patients, findings such as anorexia or weight loss, related to both systemic inflammation and aging, are frequently identified. In a meta-analysis study conducted by Verlaan et al., analyzing a total of 28 studies and 5447 malnourished elderly patients, it was reported that two out of three malnourished elderly individuals were physically frail (25). In our study, a negative correlation was found between body weight and frailty, and the BMI of the frail group was less than that of the non-frail group. Conducting further nutrition-related studies on frail COPD patients might help prevent the progression of frailty in these patients.

The TFS is in conformity with parameters such as the GOLD staging that is used to assess COPD patients, $\mathrm{BMI}$, the number of comorbidities and the drugs used, the CAT score, and the 6MWT percentage. Conventional evaluation of frailty alongside other tests for COPD assessment might hamper the progress of frailty, offer physicians supplementary information regarding COPD follow-up, and enable intervention at an early period, such as forwarding

\section{REFERENCES}

1. Lunenfeld B, Stratton P. The clinical consequences of an ageing world and preventive strategies. Best Pract Res Clin Obstet Gynaecol 2013;27(5):643-59. (PMID:23541823).

2. Chen X., Mao G., Leng S.X. Frailty syndrome: an overview. Clin Interv Aging 2014;9:433-41. (PMID:24672230).

3. Tudorache E., Fildan A.P., Frandes M., Dantes E., Tofolean D.E. Aging and extrapulmonary effects of chronic obstructive pulmonary disease. Clin Interv Aging 2017;12:1281-87. (PMID:28860729).

4. Arslan M, Meltem K.E, Sözmen $M$ et al. The Turkish adaptation of the Tilburg frailty indicator: a validity and reliability study. Turkish Journal of Geriatrics 2018;21(2):173-83. to physical and pulmonary rehabilitation, geriatric and nutritional experts to increase physical performance and quality of life of such patients.

There are many studies about frailty in elderly, or in chronic diseases. However, frailty syndrome was not studied extensively in chronic obstructive pulmonary disease, which is quite common among elderly male smokers. Moreover, the Turkish validation study of Tilburg frailty scale is published recently (4) and as far as we know, the present study is the first and only one using it to evaluate frailty in COPD patients.

Besides, there is no comprehensive study in the current literature investigating factors effective for frailty syndrome in COPD and its relationship with systemic inflammation, and the TFS test is a practical test that can be performed in a short period at the out-patient clinic. For these reasons, we believe that our study would contribute to both the medical literature and the follow-up of COPD patients.

\section{Conflicts of interest}

The authors of this article state that they have no conflict of interest.

5. Vestbo J, Hurd S.S, Agusti A.G et al. Global strategy for the diagnosis, management, and prevention of chronic obstructive pulmonary disease: GOLD executive summary. Am J Respir Crit Care Med 2013;187(4):347-65. (PMID:22878278).

6. Fabbri L.M, Luppi F, Beghe B, Rabe K.F. Complex chronic comorbidities of COPD. Eur Respir J 2008;31(1):204-12. (PMID:18166598).

7. Barnes P.J, Celli B.R. Systemic manifestations and comorbidities of COPD. Eur Respir J 2009; 33(5):1165-85. (PMID:19407051).

8. Yazici O, Tas Gulen S, Eryilmaz U, Omurlu I.K. The evaluation of cardiac functions according to chronic obstructive pulmonary disease groups. Aging Male 2020;23(2):106-11. (PMID:31037993). 
9. Kusunose M, Oga T, Nakamura S, Hasegawa $Y$, Nishimura K. Frailty and patient-reported outcomes in subjects with chronic obstructive pulmonary disease: are they independent entities? BMJ Open Respir Res 2017; 4(1): e000196. Published 2017 Jul 3. (doi:10.1136/bmjresp-2017-000196.) (PMID:28883929).

10. Limpawattana $P$, Putraveephong $S$, Inthasuwan $P$, Boonsawat W, Theerakulpisut D, Chindaprasirt J. Frailty syndrome in ambulatory patients with COPD. Int J Chron Obstruct Pulmon Dis 2017;12:1193-98. (PMID:28458530).

11. Bousquet J, Dinh-Xuan A.T, Similowski T. et al. Should we use gait speed in COPD, FEV1 in frailty and dyspnoea in both? Eur Respir J 2016;48(2):31519. (PMID:27478189).

12. Global Strategy for the Diagnosis, Management and Prevention of COPD, Global Initiative for Chronic Obstructive Lung Disease (GOLD) 2019 Report. [Internet]. Available from: https://goldcopd.org/wpcontent/uploads/2018/11/GOLD-2019-v1.7-FINAL14Nov2018-WMS.pdf. Accessed:05.10.2020.

13. Miller M.R, Crapo R, Hankinson J. et al. General considerations for lung function testing. Eur Respir J 2005;26(1):153-61. (PMID:15994402).

14. Turner G, Clegg A. British Geriatrics Society; Age U.K., Royal College of General P. Best practice guidelines for the management of frailty: a British Geriatrics Society, Age UK and Royal College of General Practitioners report. Age Ageing 2014;43(6):744-7. (PMID:25336440).

15. Guan C, Niu H. Frailty assessment in older adults with chronic obstructive respiratory diseases. Clin Interv Aging 2018;13:1513-24. (PMID:30214171).

16. lerodiakonou D, Kampouraki M, Poulonirakis I et al. Determinants of frailty in primary care patients with COPD: the Greek UNLOCK study. BMC Pulm Med 2019;19(1):63. (PMID:30876423).
17. Karadag F, Karul A.B, Cildag O, Yilmaz M, Ozcan H. Biomarkers of systemic inflammation in stable and exacerbation phases of COPD. Lung 2008;186(6):4039. (PMID:18807087).

18. Walston J, Buta B, Xue Q.L. Frailty Screening and Interventions: Considerations for Clinical Practice. Clin Geriatr Med 2018;34(1):25-38. (PMID:29129215).

19. Fried L.P, Tangen C.M, Walston J et al. Frailty in older adults: evidence for a phenotype. J Gerontol A Biol Sci Med Sci 2001;56(3):M146-56. (PMID:11253156).

20. Rockwood K, Song $X$, MacKnight $C$ et al. A global clinical measure of fitness and frailty in elderly people. CMAJ 2005;173(5):489-95. (PMID:16129869).

21. Ershler W.B, Keller E.T. Age-associated increased interleukin-6 gene expression, late-life diseases, and frailty. Annu Rev Med 2000;51:245-70. (PMID:10774463).

22. Dogrul R.T, Varan H.C, Kizilarslanoglu M.C et al. Relationship between frailty and inflammation. Eur J Geriatr Gerontol 2019;1:17-23.

23. DePew Z.S, Karpman C, Novotny P.J, Benzo R.P. Correlations between gait speed, 6-minute walk distance, physical activity, and self-efficacy in patients with severe chronic lung disease. Respir Care 2013;58(12):2113-19. (PMID:23696689).

24. Gale N.S, Albarrati A.M, Munnery M.M et al. Frailty: A global measure of the multisystem impact of COPD. Chron Respir Dis 2018;15(4):347-55. (PMID:29334783).

25. Verlaan S, Ligthart-Melis G.C, Wijers S.L.J, Cederholm T, Maier A.B, de van der Schueren M.A.E. High Prevalence of Physical Frailty Among Community-Dwelling Malnourished Older Adults-A Systematic Review and Meta-Analysis. J Am Med Dir Assoc 2017;18(5):374-82. (PMID:28238676). 